\title{
Distributed Localization in Censored Wireless Sensor Networks with Binary Data
}

\author{
Sami A. Aldalahmeh*, Rashed Alsakarnah ${ }^{\dagger}$, Saleh O. Al-Jazzar ${ }^{\ddagger}$ \\ Department of Communication and Computer Engineering \\ Al-Zaytoonah University of Jordan \\ Amman, Jordan \\ Email: *s.aldalahmeh@zuj.edu.jo, †r.alsakarnah@zuj.edu.jo, \\ $\ddagger_{\text {saleh.g@zuj.edu.jo }}$
}

\author{
Edmond Nurellari \\ College of Science, School of Engineering \\ University of Lincoln \\ Lincoln, UK \\ Email: enurellari@lincoln.ac.uk
}

\begin{abstract}
In this paper we investigate distributed localization of an intruder in wireless sensor networks (WSNs), in which the sensor nodes (SNs) censor their transmission to the fusion center (FC). The SNs locally detect the intruder and send their decision only if it is positive. The FC, on the other hand, uses those binary data to localize the intruder. We present the censored maximum likelihood (cML) localization algorithm, Furthermore, we derive two computationally simple localization algorithms, the quadratic approximate ML (QAML) and the linear approximate ML (LAML). The performance of the ML-based algorithms significantly outperforms the heuristics-based algorithms, such as the centroid method (CM) and the center of maximum enclosing rectangle (CMER), as the simulation results show.
\end{abstract}

Index Terms-Wireless sensor networks, distributed localization, censoring, binary data.

\section{INTRODUCTION}

Localization is an important application of wireless sensor networks (WSNs), especially for intrusion detection [1]. Distributed localization is well suited for WSN of the prevalent applications in WSNs, such as civilian [2], [3] and military [4] applications. However, due to limited resource nature of the WSNs [5], passive sensing is usually preferred in WSNs. In such case, the sensing modalities might be energy-based [6] or time-difference of arrival (TDOA) [7] for example. Furthermore, quantized [8] or even binary data [9] are transmitted to preserve transmission power and bandwidth.

Maximum likelihood (ML) localization of an acoustic source was studied in [10], in which energy-based analog transmitted data where used. Projection on convex sets method was adopted in [11] to localize the energy source. ML was also used in [12] to localize a diffusive source. A robust distributed detection and localization algorithm was developed in [13]. Joint detection and localization based on local decisions was considered in [14] via the generalized likelihood ratio test (GLRT). Whereas target detection and localization in clustered WSN was investigated in [15]. Quantized data were used in [16] for distributed localization in WSNs, where GLRT was adopted. Heuristic localization algorithms, which avoid complicated computation required by the ML, can be also used seamlessly with binary data. The center of maximum enclosing rectangle (CMER) and maximum enclosing circle (CMEC) al- gorithms were proposed for uniformly and randomly deployed WSNs respectively [17], [18].

In this paper, we propose the censored ML (cML) localization, which only uses positive binary decisions. However, in order to reduce the required computational complexity required by the $\mathrm{CML}$, we propose a quadratic approximate ML (QAML) and a linear approximate ML (LAML) localization algorithms, which show promising results.

The remainder of the paper is organized as follows. Section II lays down the notation used in the paper. In addition to introducing the system model adopted here. Section III presents distributed localization algorithms in censored WSNs. Simulation results are presented in Section IV. Finally, the paper is concluded by Section V.

\section{System Model}

This section firstly introduces the mathematical notion used in the paper. Then the system model of the WSN and distributed localization is presented.

Notation: Generally, upper-case symbols refer to random variables whereas lower-case symbols refer to deterministic variables. Bold notation refers to vectors. Calligraphic symbols refer to sets.

Consider a WSN consisting of $M$ of SNs uniformly deployed in a sensing field $\mathcal{F} \in \mathbb{R}^{2}$. The SNs are located on a grid in $\mathcal{F}$ with coordinates $\mathbf{x}_{i}$ for $i=1, \cdots, M$. The SNs report to a FC located at the origin without loss of generality.

The WSN is tasked with the detection of any intruders entering the sensing field. Such an intruder has a power of $P_{t}$ located at $\mathbf{x}_{t} \in \mathcal{F}$. Each $\mathrm{SN}$ samples the sensing field thus acquiring the signal [19]:

$$
\begin{aligned}
& \mathcal{H}_{0}: S_{i}=V_{i} \\
& \mathcal{H}_{1}: S_{i}=a_{i}\left(\mathbf{x}_{t}\right)+V_{i}
\end{aligned}
$$

where $\mathcal{H}_{0}, \mathcal{H}_{1}$ are the null hypothesis, alternative hypothesis respectively, and $V_{i}$ is the sensing noise, which is normally distributed with variance $\sigma_{s}^{2}$, i.e., $V_{i} \sim \mathcal{N}\left(0, \sigma_{s}^{2}\right)$ and

$$
\begin{aligned}
a_{i}\left(\mathbf{x}_{t}\right) & =\frac{\sqrt{P_{t}}}{\max \left(d_{0}, d_{i}\right)} \\
d_{i} & =\left\|\mathbf{x}_{i}-\mathbf{x}_{t}\right\|
\end{aligned}
$$


where $d_{0}$ is the SN reference distance, after which the intruder power follows the inverse-square law. Note that the sensing SNR here is defined as $\mathrm{SNR}_{s}=P_{t} / \sigma_{s}^{2}$.

Each SN locally detects the presence of the intruder via a local detector. This can be thought of as a single bit quantization of the measured data. We adopt a simple slicer as the SN's local detection defined for the $i$ th SN as

$$
I_{i}= \begin{cases}1, & S_{i} \geq \gamma \\ 0, & S_{i}<\gamma\end{cases}
$$

where $\gamma$ is the local threshold. The local detection probability at $i$ th $\mathrm{SN}$ then becomes

$$
P_{d, i}\left(\mathbf{x}_{t}\right)=\mathbb{P}\left\{I_{i}=1 ; \mathcal{H}_{1}\right\}=Q\left(\frac{\gamma-a_{i}\left(\mathbf{x}_{t}\right)}{\sigma_{s}}\right)
$$

whereas the local false alarm probability is

$$
P_{f a}=\mathbb{P}\left\{I_{i}=1 ; \mathcal{H}_{0}\right\}=Q\left(\frac{\gamma}{\sigma_{s}}\right)
$$

where $Q(\cdot)$ is the Q-function defined as

$$
Q(x)=\int_{x}^{\infty} e^{-\frac{t^{2}}{2}} d t
$$

Although the detection and false alarm probabilities defined in (6) and (7) are usually used in clairvoyant detectors, in which the intruder's parameters are assumed to be known, they are also applicable in the case they are not [16]. Where an energy detector is used to produce the data in (1) and (2). The central limit theorem guarantees the Gaussian distribution of the collected data when the number of samples is adequately large.

The SNs censor there transmission in order to save power. Only the positive decision is sent to the $\mathrm{CH}$. To do so, the SNs use on-off-keying (OOK) to send their decisions to the FC. The communication channel is assumed to be ideal. The received binary data at the FC is used to localize the intruder. Note, however, that some SNs might falsely detect an intruder under $\mathcal{H}_{1}$, which is known as the spurious detection problem [20]. This occurs in far SNs from the intruder, where the sensed signal is weak and is dominated by noise that triggers the false alarm.

\section{Distributed LOCALIZATION With BinARY DATA}

In this section we discuss the maximum likelihood estimation (MLE) of the intruder's location using the complete and censored binary decisions. Then we introduce the approximated censored MLE algorithm.

\section{A. Maximum Likelihood Estimation}

Firstly, we present MLE of the intruder's location using all the available binary data, i.e., positive and negative local decisions. This will serve as a benchmark for the other algorithms.

The likelihood function under $\mathcal{H}_{1}$ of the all the local decisions is given by

$$
l\left(I_{0}, \cdots, I_{M}\right)=\prod_{i=1}^{M} P_{d, i}\left(\mathbf{x}_{t}\right)^{I_{i}}\left(1-P_{d, i}\left(\mathbf{x}_{t}\right)\right)^{\left(1-I_{i}\right)}
$$

and consequently the log-likelihood function (LLF) reads as

$$
\begin{aligned}
L_{M L}\left(\mathbf{x}_{t}\right) & =\log l\left(I_{0}, \cdots, I_{M}\right) \\
& =\sum_{i=1}^{M} I_{i} \log P_{d, i}\left(\mathbf{x}_{t}\right)+\left(1-I_{i}\right) \log \left(1-P_{d, i}\left(\mathbf{x}_{t}\right)\right) .
\end{aligned}
$$

Note that the second term in the summation above represents the miss-detection occurring in the SNs. However, this cost function is highly nonlinear and non-convex as evident in Fig. 1a. The intruder is localized by finding the maximizing the the log-likelihood function in (10) with respect to $\mathbf{x}_{t}$. i.e.,

$$
\begin{aligned}
\hat{\mathbf{x}}_{t, M L} & =\arg \max _{\mathbf{x}_{t}} \sum_{i=1}^{M} I_{i} \log P_{d, i}\left(\mathbf{x}_{t}\right) \\
& +\left(1-I_{i}\right) \log \left(1-P_{d, i}\left(\mathbf{x}_{t}\right)\right) .
\end{aligned}
$$

It is clear, however, that the above problem is computationally and numerically challenging, due to having many local minimums and maximums.

Recall that in censored WSN only SNs with positive local decisions send their data to the FC. As such only the first term in (11) is retained. Then the log-likelihood function in the censored ML (cML) is simpler and has the form

$$
L_{c M L}\left(\mathbf{x}_{t}\right)=\sum_{i=1}^{M} I_{i} \log P_{d, i}\left(\mathbf{x}_{t}\right)
$$

Fig. 1b shows the corresponding log-likelihood function, which is obviously smoother and hence simpler to handle in terms of solving the below optimization problem

$$
\hat{\mathbf{x}}_{t, c M L}=\arg \max _{\mathbf{x}_{t}} \sum_{i=1}^{M} I_{i} \log P_{d, i}\left(\mathbf{x}_{t}\right) .
$$

\section{B. Approximated cMLE}

The cMLE problem in (13) is still highly nonlinear, which becomes evident when closely examining the term

$$
\log P_{d, i}\left(\mathbf{x}_{t}\right)=\log Q\left(\frac{\gamma}{\sigma_{s}}-\frac{a_{i}\left(\mathbf{x}_{t}\right)}{\sigma_{s}}\right)
$$

As such, we resort to approximating the term in (14) by recalling that the Q-function can be approximated by the following Chernoff upper bound

$$
Q(x) \approx e^{-\frac{x^{2}}{2}}
$$

When instrumenting this approximation, we are able to develop a simpler form of the censored log-likelihood in (12), which we name as the quadratic approximated ML follows

$$
\begin{aligned}
L_{Q A M L}\left(\mathbf{x}_{t}\right) & =-\frac{1}{2 \sigma_{s}^{2}} \sum_{i=1}^{M} I_{i}\left(\gamma-a_{i}\left(\mathbf{x}_{t}\right)\right)^{2} \\
& =-\frac{1}{2 \sigma_{s}^{2}} \sum_{i=1}^{M} I_{i}\left(\gamma^{2}-2 \gamma a_{i}\left(\mathbf{x}_{t}\right)+a_{i}^{2}\left(\mathbf{x}_{t}\right)\right)
\end{aligned}
$$




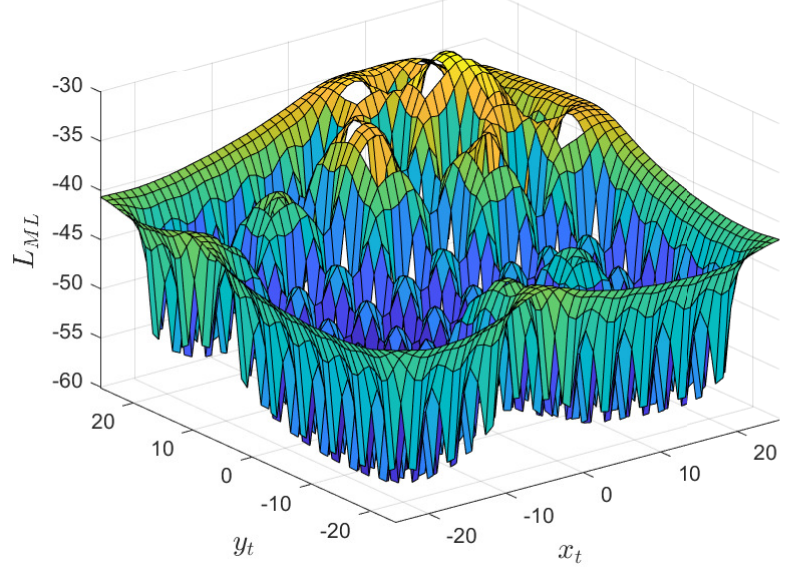

(a) ML localization.

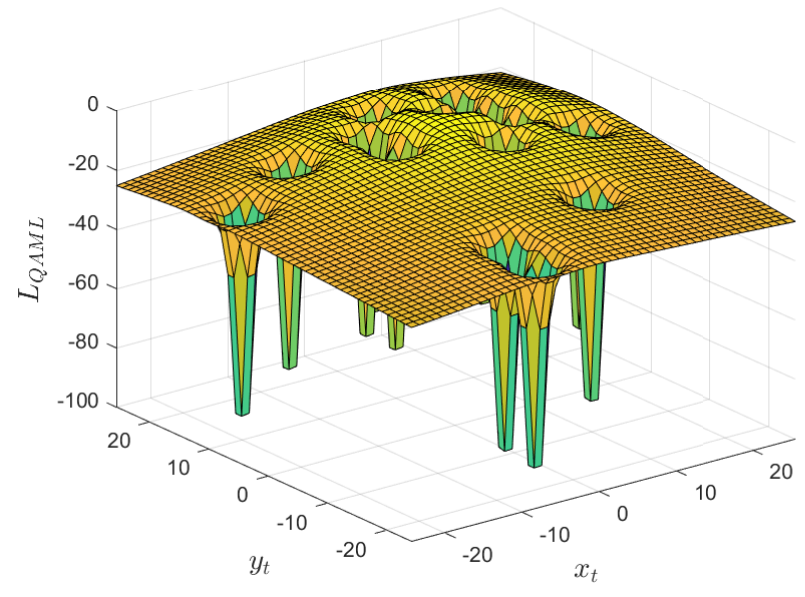

(c) Quadratic approximated cML localization.

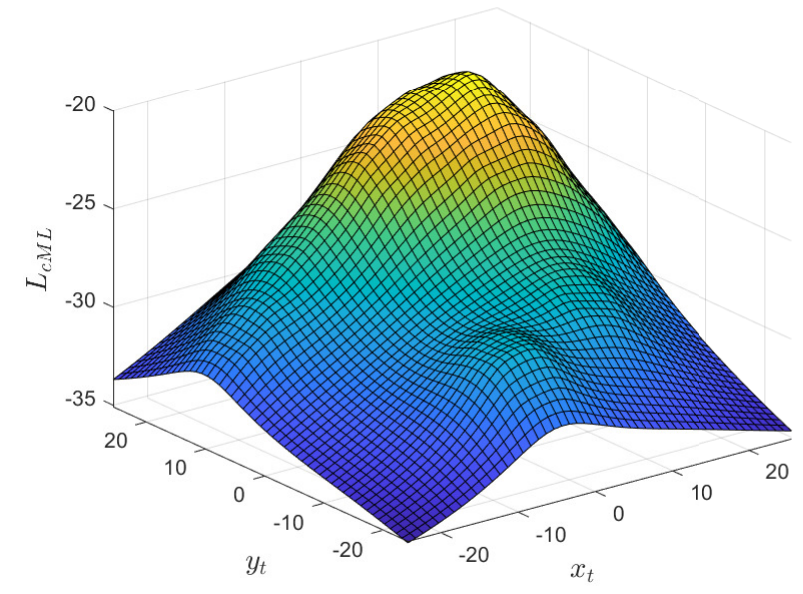

(b) Censored ML localization.

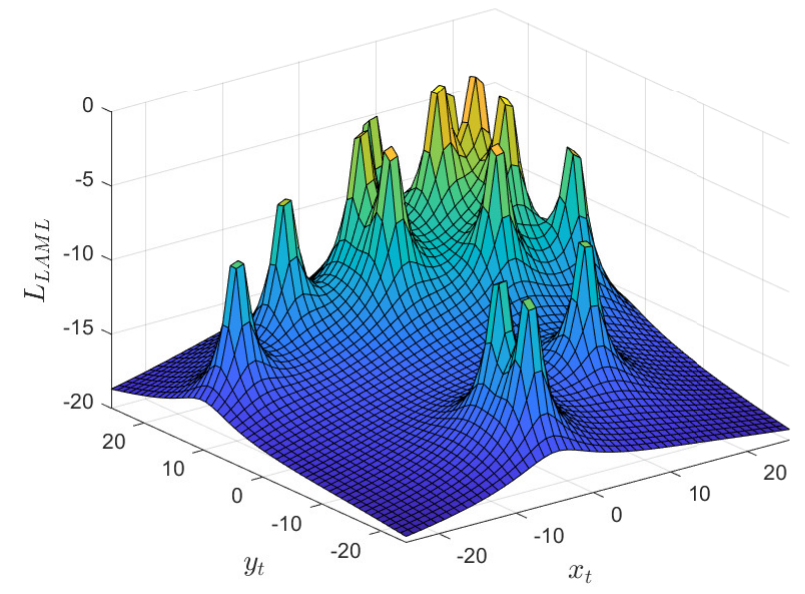

(d) Linear approximated cML localization.

Fig. 1: Log-likelihood functions for the ML-based localization algorithms for $81 \mathrm{SNs}$, intruder at $\mathbf{x}_{t}=(12,15)$, sensing SNR $\mathrm{SNR}_{s}=20 \mathrm{~dB}, P_{f a}=0.05$.

Note that the resulting maximization problem can be formulated as

$$
\hat{\mathbf{x}}_{t, Q A M L}=\arg \max _{\mathbf{x}_{t}} \sum_{i=1}^{M} I_{i}\left(2 \gamma a_{i}\left(\mathbf{x}_{t}\right)-a_{i}^{2}\left(\mathbf{x}_{t}\right)\right)
$$

since the constant $\gamma^{2}$ in the summation in (16) can be ignored.

The LLF is shown in Fig. 1c where it contains several local minimums located at the detecting SNs, in contrast to the ML LLF in (10). Nonetheless the cML LLF is smoother than the QAML and hence it is expected to have a better performance.

The deep minimums in Fig. 1c can be explained by the quadratic behaviour of the cost function that emphasizes large values. To mitigate this effect we propose using a linear function instead of the quadratic one, leading to the linearly approximated ML (LAML) cost function written as

$$
L_{L A M L}\left(\mathbf{x}_{t}\right)=\sum_{i=1}^{M} I_{i}\left(a_{i}\left(\mathbf{x}_{t}\right)-\gamma\right)
$$

As expected, Fig. 1d shows shallower maximums and hence better localization performance achieved by solving the following optimization problem

$$
\hat{\mathbf{x}}_{t, L A M L}=\arg \max _{\mathbf{x}_{t}} \sum_{i=1}^{M} I_{i}\left(a_{i}\left(\mathbf{x}_{t}\right)-\gamma\right)
$$

\section{Heuristic Localization Algorithms}

As noticed above, MLE-based localization algorithms are relatively complicated. Hence, several heuristic localization algorithms have been suggested. We briefly discuss the centroid 


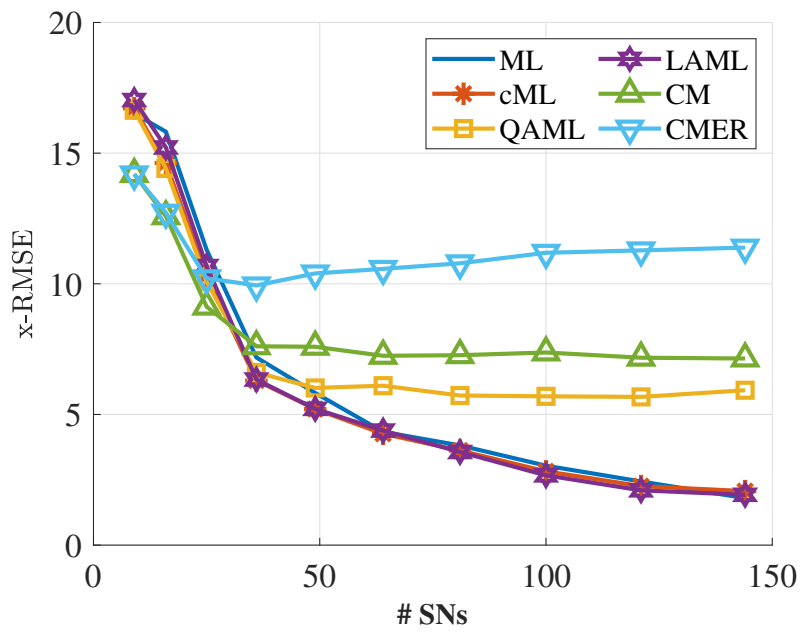

(a) X-coordinate.

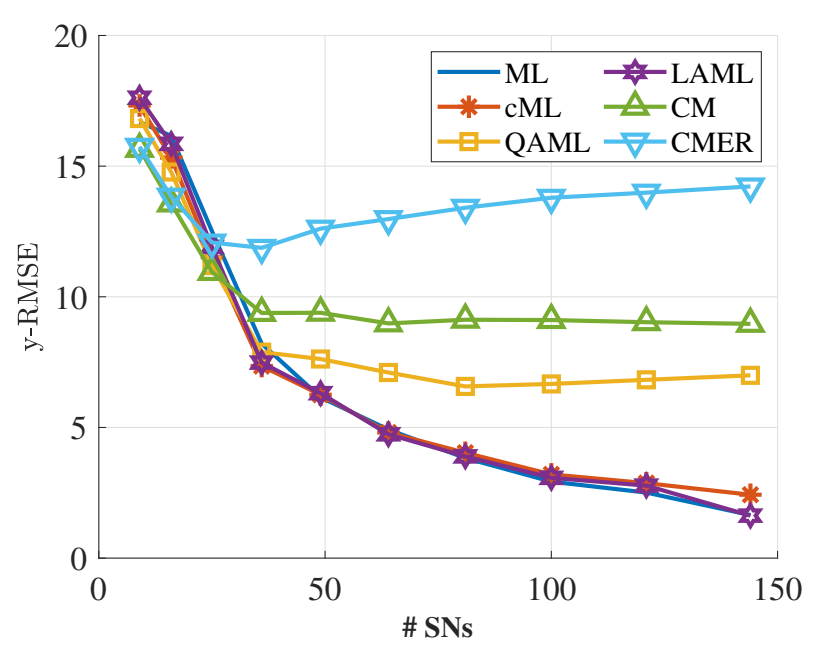

(b) y-coordinate.

Fig. 2: Root mean square errors for an intruder at $\mathbf{x}_{t}=(12,15)$, sensing $\mathrm{SNR}^{\mathrm{SNR}} \mathrm{SN}_{s}=20 \mathrm{~dB}, P_{f a}=0.05$.

method $(\mathrm{CM})$ and the center of maximum enclosing rectangle method (CMER).

1) CM Localization: The CM localization estimates the intruder's location by simply computing the mean coordinates of the detecting SNs. i.e.,

$$
\hat{\mathbf{x}}_{t, C M}=\frac{\sum_{i=1}^{M} I_{i} \mathbf{x}_{i}}{\sum_{i=1}^{M} I_{i}}
$$

2) CMER Localization: The CMER is implemented in a uniformly deployed WSN. The intruder's position is estimated as the center of a rectangle that encloses the farthest detecting SNs.

However, both methods are sensitive to the spurious detection problem since the falsely detecting SNs would bias the estimate.

\section{Simulations and Performance Analysis}

We simulate a WSN in a field of $50 \times 50$. The intruder position $\left(\mathrm{x}_{t}\right)$ is located at $(12,15)$ units. It has a power of $P_{t}=10$ and the sensing SNR $\left(\mathrm{SNR}_{s}\right)$ is $20 \mathrm{~dB}$. The sensing reference distance is set to $d_{0}=1$ unit. The local probability of false alarm $\left(P_{f a}\right)$ is 0.05 . The WSN is simulated for 1000 Monte Carlo iterations. We simulate the following localization algorithms: ML in (13), censored ML (cML) in (13), QAML in (17), LAML in (19), the CM in (20) and the CMER method. Note that the solutions for QAML and LAML were obtained through grid search, in which the field was divided into 50 square grids and the estimate is considered to the the grid's center.

Fig. 2 shows the root mean square error for intruder's localization as the number of the SNs in the network increases. As predicted earlier, the ML is performance slightly worse than the cML. Interestingly however, the LAML performance is almost similar to theat of the optimal ML and the cML. On the other hand, the QAML performance better than the CM and CMER but not as good as the other ML-based algorithms, due to the quadratic behaviour as explained earlier. Another interesting note that the heuristic algorithms' performance do not improve (or worsen in the case of CMER) when the number of SNs increases, in contrast to the ML-based algorithms, except for the QAML. This is attributed to the fact that having more SNs implies having more spurious detection and hence more estimation bias. Similarly, the QAML seems to be sensitive to the spurious detection problem.

On the other hand, Fig. 3 demonstrates the localization performance as a function of the local probability of false alarm, $P_{f a}$. Recall that the local detection threshold is directly determined by $P_{f a}$. It is clear that heuristics-based algorithms are negatively affected when $P_{f a}$ increases since spurious detection increases consequently. In contrast, ML-based algorithms do not show significant change in performance as $P_{f a}$ changes. Although an optimal $P_{f a}$ is noticed. Except in the case of the QAML algorithm that behaves similar to the CM and CMER counterparts. This might be explained by the numerical sensitivity inherit in the quadratic nature of the algorithm, which leads to the noticed behaviour.

\section{CONCLUSiOnS AND Future WORK}

We have studied distributed localization in censored WSN using binary transmission. We have derived the censored maximum likelihood (cML) localization algorithm, in addition to two approximated versions, the quadratic approximated ML (QAML) and the linearly approximated ML (LAML) algorithms, which are simpler computationally. It has been shown that the cML localization performance is almost reaches the optimal ML performance and so does the LAML when increasing the number of SNs. Moreover, the ML-based algorithms are almost insensitive to changes in the local detection performance (except the QAML), in contrast to the heuristicsbased counterparts. In future works, intruder localization will 


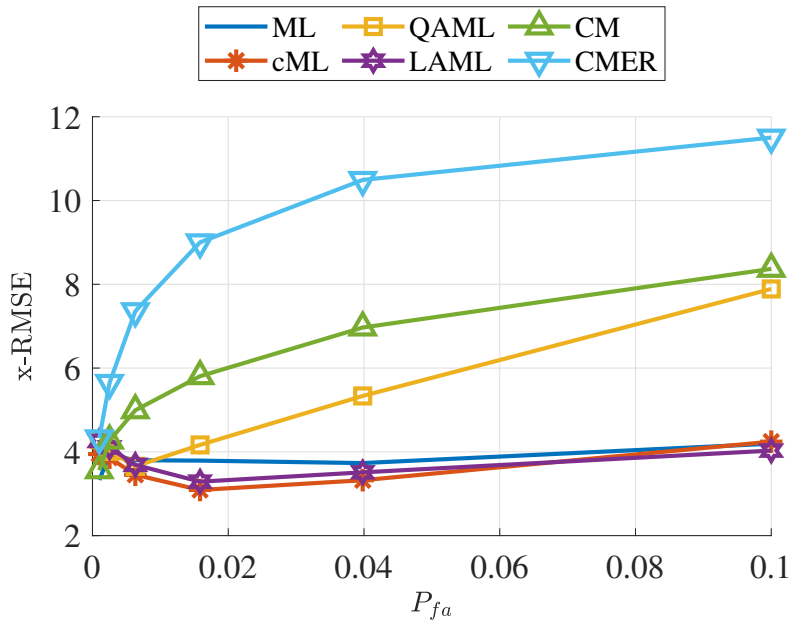

(a) x-coordinate.

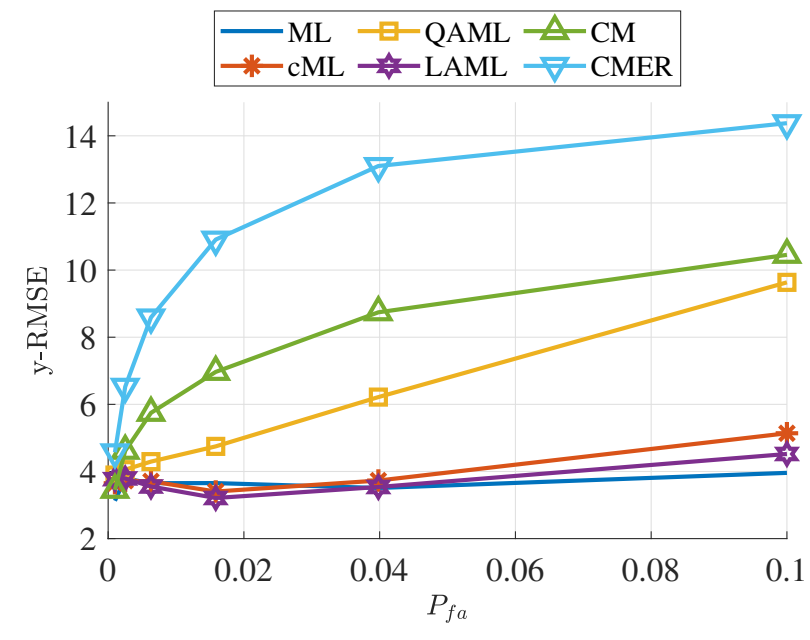

(b) y-coordinate.

Fig. 3: Root mean square errors for $81 \mathrm{SNs}$, intruder at $\mathbf{x}_{t}=(12,15)$ and sensing $\mathrm{SNR}^{\mathrm{SNR}} \mathrm{SN}_{s}=20 \mathrm{~dB}$.

be considered for randomly deployed WSNs. In addition to investigating potential power savings in the WSN as a consequence of the optimally choosing the local detection threshold.

\section{ACKNOWLEDGEMENT}

This work was supported by the Al-Zaytoonah University Research Grant 12/11/2020-2021.

\section{REFERENCES}

[1] U. Nazir, N. Shahid, M. A. Arshad, and S. H. Raza, "Classification of localization algorithms for wireless sensor network: A survey," in 2012 International Conference on Open Source Systems and Technologies, Dec 2012, pp. 1-5.

[2] Bushra Rashid and Mubashir Husain Rehmani, "Applications of wireless sensor networks for urban areas: A survey," Journal of Network and Computer Applications, vol. 60, pp. 192-219, 2016.

[3] Frank Stajano, Neil Hoult, Ian Wassell, Peter Bennett, Campbell Middleton, and Kenichi Soga, "Smart bridges, smart tunnels: Transforming wireless sensor networks from research prototypes into robust engineering infrastructure," Ad Hoc Networks, vol. 8, no. 8, pp. 872 - 888, 2010.

[4] Zhi Sun, Pu Wang, Mehmet C Vuran, Mznah A Al-Rodhaan, Abdullah M Al-Dhelaan, and Ian F Akyildiz, "Bordersense: Border patro through advanced wireless sensor networks," Ad Hoc Networks, vol. 9, no. 3, pp. 468-477, 2011.

[5] Chee Yee Chong and S. P. Kumar, "Sensor networks: evolution, opportunities, and challenges," Proceedings of the IEEE, vol. 91, no. 8, pp. 1247-1256, 2003.

[6] Dan Li and Yu Hen Hu, "Energy-based collaborative source localization using acoustic microsensor array," EURASIP J. Appl. Signal Process., vol. 2003, pp. 321-337, 2003.

[7] Enyang Xu, Zhi Ding, and S. Dasgupta, "Robust and low complexity source localization in wireless sensor networks using time difference of arrival measurement," in Wireless Communications and Networking Conference (WCNC), 2010 IEEE, apr. 2010, pp. 1 -5.

[8] Yoon Hak Kim and A. Ortega, "Quantizer design for source localization in sensor networks," in Proceedings. (ICASSP '05). IEEE International Conference on Acoustics, Speech, and Signal Processing, 2005., March 2005, vol. 4, pp. iv/857-iv/860 Vol. 4

[9] Zhi-Quan Luo, "Universal decentralized estimation in a bandwidth constrained sensor network," Information Theory, IEEE Transactions on, vol. 51, no. 6, pp. 2210-2219, June 2005.
[10] Xiaohong Sheng and Yu-Hen Hu, "Maximum likelihood multiple-source localization using acoustic energy measurements with wireless sensor networks," Signal Processing, IEEE Transactions on, vol. 53, no. 1, pp. $44-53$, jan. 2005.

[11] D. Blatt and A.O. Hero, "Energy-based sensor network source localization via projection onto convex sets," Signal Processing, IEEE Transactions on, vol. 54, no. 9, pp. $3614-3619$, sep. 2006.

[12] Saravanan Vijayakumaran, Yoav Levinbook, and Tan F. Wong, "Maximum likelihood localization of a diffusive point source using binary observations," Signal Processing, IEEE Transactions on, vol. 55, no. 2, pp. 665-676, 2007.

[13] S. Aldalahmeh and M. Ghogho, "Robust distributed detection, localization, and estimation of a diffusive target in clustered wireless sensor networks," in International Conference on Acoustics Speech and Signal Processing (ICASSP) 2011, Prague, Czech Republic, May 2011, IEEE.

[14] R. Niu and P. K. Varshney, "Joint detection and localization in senso networks based on local decisions," in Signals, Systems and Computers, 2006. ACSSC '06. Fortieth Asilomar Conference on, Nov. 2006, pp. 525 -529 .

[15] Sami A. Aldalahmeh, Mounir Ghogho, Des McLernon, and Edmond Nurellari, "Optimal fusion rule for distributed detection in clustered wireless sensor networks," EURASIP Journal on Advances in Signal Processing, vol. 2016, no. 1, pp. 5, Jan 2016.

[16] Ruixin Niu and Pramod K. Varshney, "Target location estimation in sensor networks with quantized data," IEEE Transactions on Signal Processing, vol. 54, no. 12, pp. 4519-4528, Dec 2006.

[17] Arian Shoari and Alireza Seyedi, "Localization of an uncooperative target with binary observations," in 2010 IEEE 11th International Workshop on Signal Processing Advances in Wireless Communications (SPAWC), June 2010, pp. 1-5.

[18] Arian Shoari, Gonzalo Mateos, and Alireza Seyedi, "Analysis of target localization with ideal binary detectors via likelihood function smoothing," IEEE Signal Processing Letters, vol. 23, no. 5, pp. 737741, May 2016.

[19] R. Niu and P. K. Varshney, "Performance analysis of distributed detection in a random sensor field," Signal Processing, IEEE Transactions on, vol. 56, no. 1, pp. $339-349$, Jan. 2008.

[20] M. Guerriero, L. Svensson, and P. Willett, "Bayesian data fusion for distributed target detection in sensor networks," Signal Processing, IEEE Transactions on, vol. 58, no. 6, pp. $3417-3421$, June 2010. 